\title{
Terrestrial communications system design and MOE factors*
}

\author{
Young C. Park ${ }^{\dagger}$ \\ Department of Information and Communication Engineering, Baekseok University, Cheonan, Korea
}

(Received December 12 2007, Accepted March 18 2008)

\begin{abstract}
An area network contains significant redundancy and can sustain considerable damage, or cope with substantial movement, because of the alternative routes available. In this paper we examine some measure of effectiveness (MOE) factors for Army Terrestrial Communications System (TCS) in detail: mobility, security/survivability, system availability, grade of service (GOS), design flexibility, interoperability, reliability, maintainability, cost effectiveness, and enhanceability. We also shall analyze the average link delay versus network size and arrival rate which is related to GOS factors. We believe that the flood search routing algorithm provides survivability and is a viable option for providing a reliable routing framework in Army Terrestrial Communications System.
\end{abstract}

Keywords: measure of effectiveness(MOE), grade of service(GOS), flood search routing, survivability

\section{Introduction}

Humans have long dreamed of possessing the capability to communicate with each other anytime, anywhere. As the twenty-first century approaches, technical capabilities have become so sophisticated that stationary telephones, facsimile machines, computers, and other communications devices-connected by wires to power sources and telecommunications network-are almost ubiquitous in many industrialized countries. Success on the battlefield depends to a large extent on the timely receipt of accurate information presented in a format that can be digested readily by the commander and staff to allow them to prepare appropriate plans. And modern armies are entering a period of sustained and substantial change in structure, doctrine and use of technology. In the face of such change, communications requirements must be reassessed through the development of a comprehensive, robust, flexible architectural framework for modern tactical communications systems. The tactical level units are tasked with missions requiring entirely different communication systems. In the tactical communications peer-to-peer nodes must remain at top priority to provide survivable communications $^{[11]}$. On the future battlefield it is essential that commanders can command and control on the move. This includes continuity of command and control whilst deploying from the barracks as well as whilst moving around the battle-space. A commander should be able to control force elements regardless of location. Two distinct battlefield communications systems have developed to support the tactical commander. The first, above battalion, required high capacity links provided by the formation to interlink supported units with headquarters. The links were usually duplex and were limited to be from one unit to another. These types of infrastructure links became known as trunk communications in line with their commercial equivalents. The second type of communications developed to allow units at battalion and below to perform tactical tasks. These links were flexible and responsive, under the direct control of the commander. Links were established using single-frequency, half-duplex, all-informed radio nets allowing the commander maximum flexibility

\footnotetext{
* The author wishes to thank Dr. Jiuping Xu and the anonymous reviewers for their suggestions in improving the earlier version of this paper presented in the IEEE international conference on Industrial engineering and Engineering management (IEEM 2007).

† Corresponding author. Tel: +82415502764; E-mail address: ycpark@bu.ac.kr.
} 
to command a number of subunits. These types of communications have become known as single channel radio. An area network contains significant redundancy and can sustain considerable damage, or cope with substantial movement, because of the alternative routes available. A key advantage of this network topology is its capability to automatically cope with traffic routing changes resulting from movement of subscribers or network outages. Communication nodes can be moved rapidly to reconfigure the network as required by the tactical situation. Commanders are no longer constrained by their communications links and can deploy as required by the tactical situation with the only siting constraint that the headquarters location must be able to communicate to at least one of the trunk nodes.

For the past years in many NATO participating countries projects on developing of new generation tactical communication systems have been carried out. Some of these projects are TACOMS-Post 2000 (TP2K: Tactical Communications Systems for the Land Combat Zone Post-2000) - NATO, WIN-T (War-fighter Information Network-Tactical) - USA, RITA 2000- France and FALCON/CORMORANT- Great Britain. These tactical communication networks are very similar to mobile ad hoc networks which are characterized by dynamic topologies, bandwidth-constraints, variable capacity links, limited physical security and high degree of survivability ${ }^{[5]}$. The WIN-T is US Army XXI's tactical telecommunications system consisting of communication infrastructure and network components from the maneuver battalion to the theater rear boundary. The WIN-T network provides command, control, communications, computers, intelligence, surveillance and reconnaissance (C4ISR) support capabilities that are mobile, secure, survivable, seamless, and capable of supporting multimedia tactical information systems within the warfighters' battle-space. WIN-T will be the integrating communications network for the Objective Force (OF), optimized for offensive and joint operations, while providing the Theater Commander in Chief (CINC) the capability to perform multiple missions simultaneously with campaign quality. WIN-T is the OF high-speed and high capacity backbone communications network ${ }^{[1]}$.

At the heart of the future battlefield integrated information system will be architecture of layered communications composed three distinct sub-networks: localized area networks, wide area networks and the range extension network. The network systems will be used to provide integration of grossly dissimilar transmission media with different characteristics and functions to exploit state-of-art advances and emerging multi-media distribution technologies. ROKA (Republic of Korea Army) has developed the area and range extension networks, Terrestrial Communications System (TCS) for the battlefield communication.

This paper organized as follows. Following this introduction, we introduce design considerations for tactical communications system and MOE factors for the provision of tactical communications in section 2. In section 3, routing algorithm and Terrestrial Communications System (TCS) architecture for Army is described. In section 4, modeling and simulation results are presented. Finally, conclusions are presented in section 5 .

\section{Design considerations and MOE factors}

Army communications are primarily surface based and constrained by limited bandwidth, limited ability to operate on-the-move (OTM), inadequate interoperability, and susceptibility to line-of-sight (LOS) obstructions. Because of these constraints, existing communications systems are unable to support the information pull required by warfighters of the next decades. This information pull requires global access to large volumes of intelligence data. New technologies will be required to meet the need for real-time, multimedia, wideband width communications. The additional requirement that continuity of operations and capacity of new communications services not be limited by terrain or sender/receiver platform motion reduces the number of potential technology solutions.

There is no universal agreement on the meaning of MOE although Sproles has suggested a definition for Measures of Effectiveness ${ }^{[10]}$. This was arrived at by not only using dictionary definitions of the components of the expression but by drawing upon the thread of agreement that can be found in the literature when the term is used. The suggested definition is that MOEs are

'... standards against which the capability of a solution to meet the needs of a problem may be judged. The standards are specific properties that any potential solution must exhibit to some extent ...' 
A number of measure of effectiveness (MOE) factors are considered for the provision of tactical communications. In this paper we examine some MOE factors in detail: mobility, security/survivability, system availability, grade of service (GOS), design flexibility, interoperability, reliability, maintainability, cost effectiveness, and enhanceability ${ }^{[10,12]}$.

The first factor, mobility, is of particular significance in the mountainous environment like Korea in which the equipment will be expected to operate. Problems of mobility in the operation of the network also apply to subscribers, switches, and other support equipment such as transmission equipments and generators. Constraints on the mobility of the physical equipment are relatively obvious. Small physical size and weight is advantageous in terms of transportation over difficult terrain and in the equipment concealment. Low power consumption enables smaller generators to be used and consequently less fuel needs to be carried. Less power, generally also results in lower operating temperatures and therefore reduced infra red signature and reduced detection probability. And the frequent movement of equipment necessitates a high degree of flexibility in configuration. In a hostile mobile environment, the equipment must be capable of being put quickly into and out of service as the situation develops. Fast set up and tear down times are therefore of importance in the tactical communications system. The key to the application of the equipment is the use of mobile circuit switches which are housed in a rugged container suitable for installation in small combat vehicles, 5/4 ton trucks. The switch weighs only $70 \mathrm{Kg}$ 's and has a power consumption of less than 90 watts. Many of the mobility factors considered are directly related to the equipment engineering. There are also wider system level implications on the communication network.

Static subscribers gain access to the switches either by direct connection or by the use of multiplexers. Mobility in the network means that it may be necessary for the switch providing services to change. This means that the new switch must 'accept' the subscribers, and the old switch must 'forget' them. Any calls to subscribers in the network must be routed to the correct switch. Mobile subscribers must be given facilities to allow them to roam in a secure mode, whilst in connection with the network. The activity of 'accepting' and 'forgetting', and correct routing are also necessary for mobile subscribers. To provide an area coverage, this process must be fully automatic, and to satisfy this objective a highly adaptive scheme has been proposed.

The provision of objective methods of providing communications network security is a second key area for system performance assessment. The use of encryption techniques is of major importance in assuring the overall security of all types of communication. In addition to the benefits provided by making it impossible for unauthorized monitoring of radio links to determine what information is being passed over that link, encryption also provides traffic flow security by making it impossible to detect the number of calls in progress over a link. Such information, were it available, could provide an indication of areas of high military activity. The design of the equipment must also take account of the wider aspects of communication security - COMSEC. In this respect it must be designed to minimize electrical emanation and must have the necessary in built controls to limit unauthorized access. The security of the network and hence its survivability are also enhanced by the capability for frequent and rapid deployment and concealment from the enemy ${ }^{[9]}$.

The modern battlefield represents a harsh electromagnetic environment within which the tactical communications system must survive. The security of the network and hence its survivability are also enhanced by the capability for frequent and rapid deployment and concealment from the enemy. The bulk encryption devices provide a cost effective method and highly secure solution. Whenever radio links are employed between switches, or from remote multiplexed subscriber communities, then the use of bulk encryption equipment is considered. A communication system is survivable if it has: sufficient capacity to handle traffic levels, an ability to manage existing capability through techniques such as dynamic bandwidth management, the necessary levels of security, low probability of intercept, resistance to jamming and interference, mobility, alternative routing, redundancy, and sufficient reserves.

Another indicator of the networks ability to satisfy the communication needs is system availability. System availability must be maintained particularly for the key network subscribers. Of interest here, are the methods by which it is possible to maintain facilities even when the network has been subjected to damage. If the communication network comes under attack and damage is sustained then redundancy built into the network provides a number of routes between any two points. All switches in the network are automatically updated as the network connectivity changes and are able to reroute calls via alternative paths. If substantial 
damage does occur, the remaining resources may not be able to provide the same grade of service (GOS) to all users. For this reason the use of pre-emption facilities for priority calls will always ensure that free channels are made available to important subscribers. The network is therefore able to make maximum utilization of transmission, whilst providing the high degree of flexibility even under heavy damage scenarios. The GOS is affected by four main components: the ability of subscribers to originate calls, the ability of subscribers to receive calls, the life of the information, and the ability of the network to provide a connection. One of the GOS factors is link delay between two nodes and we shall analyze it in section IV.

Operational flexibility is also an essential requirement of the tactical communications system. To maximize the retention of existing inventory, the capability to interface to existing equipment is required. Flexibility in the use of transmission resources enables the best media to be chosen to ideally match the deployment scenario and the local propagation conditions.

The systems and networks within the tactical communications system must be interoperable with other tactical networks, strategic networks, unclassified commercial networks, as well as networks and systems of other services and allies. This interoperability is essential if information is to be able to flow seamlessly between any two points in the battle-space or between any point in the battle-space and the strategic communications system.

The MOE factor relating to reliability and maintainability of the system is necessary, not only, to ensure continuity of service, but also to achieve economic operation. Reliability leads to cheaper operation since less spares are required to user confidence in the system and to increased availability of network resources. The objectives of maintainability are based around the simplicity of maintenance and the ease of fault diagnosis and repair. All the equipment makes extensive use of Built in Test Equipment (BITE). This provides a speedy and efficient means of checkout and identification of faults down to module level.

When considering potential solutions of building tactical communication systems, it is also necessary to take the cost factor into account. Cost effectiveness must be accessed against not only the acquisition and life support costs but also against the potential benefits to be derived from the solution.

The last MOE factor to be considered is the capability of the system to be enhanced. The tactical communications system should be able to evolve as part of an overall $\mathrm{C}^{4} \mathrm{I}$ (command, control, communication, computing and intelligence) program.

\section{Routing algorithm and terrestrial communications system architecture}

Radio communications technology is widely used by military units at all levels. The ideal tactical communications system architecture would provide a mobile infrastructure to support mobile users. It would therefore be a single homogeneous network supporting all communicating entities in the battle-space. The Army's Terrestrial Communications System (TCS) is a communications network of radio stations, normally carried on 5/4 ton military vehicles, linked by a wire network for communications between the more static headquarters. Communications may be by voice, facsimile, data or image. The TCS provides circuit-switched secure digital voice and data communications for a nominal corps area and three associated divisions. It also provides packet-switched data communications service for local area network (LAN) and individual hosts. The nominal area coverage network consists of 32 communication nodes. A block diagram of TCS is shown in Fig. 1. The Extension Node (EN) units extend the switching capability of the Node Controller (NC) via VHF (very high frequency) Line of Sight (LOS) radios. A normal NC consists of a digital automatic switch installation and an automatic radio integration interface with a manned hybrid switch and a manned radio integration capability. Routing algorithms of the switch choose a hop-by-hop path based on information about link connectivity. The simplest scheme is flood search routing, in which a packet is transmitted on all links from the source to neighboring nodes, which then repeat the process ${ }^{[6]}$. The identity of a subscriber is stored at the node to which he is attached. When a call is made to him from a subscriber at a different switch, and his location is not known, a flood search message is sent out. This propagates to every node in the network and the node that recognizes the required directory number respond with its own identity. The path taken by the search message and its reply is not significant so no channels are reserved and no memory of the path is retained. The original node is therefore aware of the required destination node before the traffic path is 
requested. Each node maintains an updated record of network connectivity data from which it derives a route preference table giving the preferred and alternative exit links to every other node in the network. From this table, and taking into account the current traffic loading, the originating node selects a link to the next node on which to route a call. The node receiving the message recalculates the next preferred link, again taking into account the local traffic loading. This procedure, known as delegated routing is followed for each node until the route is established between the originating and destination nodes. This technique is highly adaptive to change in the network connectivity caused by reconfiguration, temporary congestion or damage. The process is also carried out automatically at high speed and is therefore transparent to the subscriber. At the originating node the association between the called subscriber and his location is retained on a frequency of use basis. If the called subscriber is wanted again within a reasonable period, the flood search will only be required if he has moved to a new node. This routing method has been developed to overcome the disadvantage of the use of a flood search for every trunk call. Complete reliance on flood search could result in overload and uneconomic increases in the provision of signaling channels. Flooding is inefficient but can be the best strategy when a network topology changes rapidly. In general, network topologies vary rapidly in mobile packet radio networks, with links constantly being lost and new ones established. Therefore, the network management component needs to disseminate connectivity information more rapidly than is necessary in wired networks.

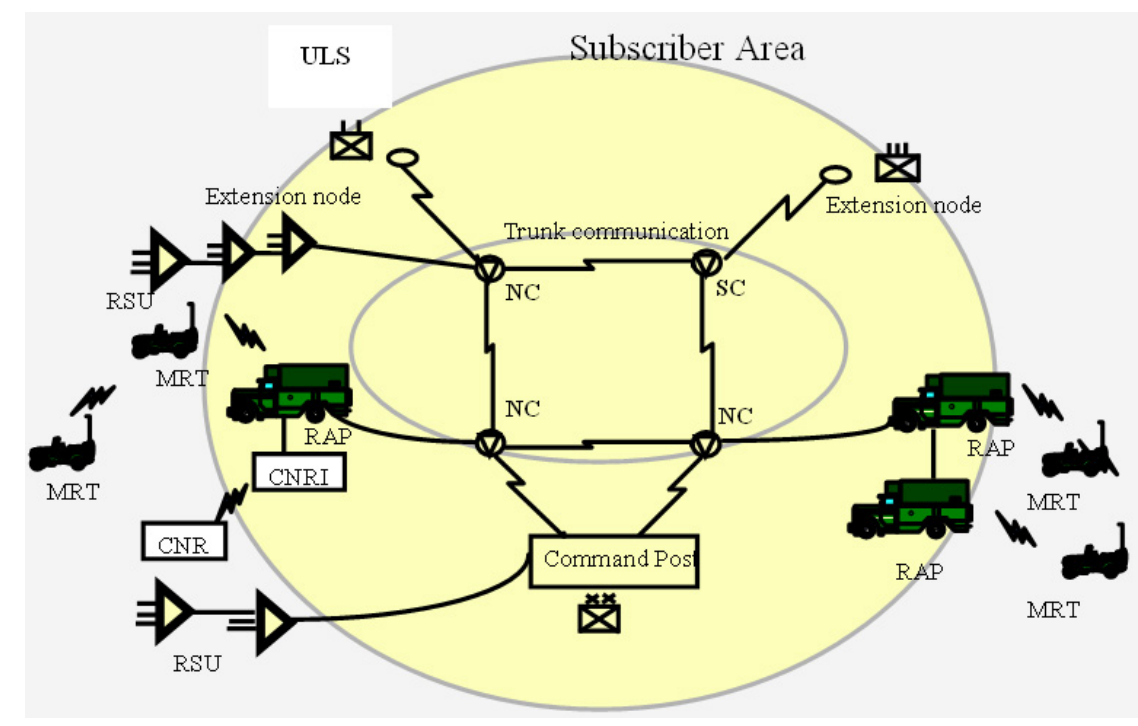

Fig. 1. TCS Architecture

The NC Shelter is configured into four shelters: Switching Shelter, Large Extension Node Shelter, Radio Access Point (RAP) Shelter and Unit Level Switch (ULS) Shelter. The System Controller (SC) communicates with NCs and provides an automatic capability for planning and managing the TCS. The SC Shelter is configured into three shelters: Planning Shelter, Management Shelter and Maintenance Shelter. Each shelter carries two 10kW trailer mounted gasoline generators. And the RAP and the ULS Shelters can be added if necessary. Subscribers are wired to the EN shelters. The Radio Access Points (RAPs) is an automatic interface between the mobile subscribers with Mobile Radio Terminals (MRTs) and the network. The Combat Net Radio (CNR) can be interfaced with RAP via Combat Net Radio Interface (CNRI) unit. A small group of users up to 30 subscribers are accessed to the network via The Remote Switching Unit (RSU) with two 256 kbps digital trunk group ${ }^{[4]}$.

A typical Unit Level Switch (ULS) for small group is shown in Fig. 2. Personal computers can be accessed via Digital Multi Role Terminal (DMT) to RSU. The radio equipment utilized for the Down the Hill (DTH) application of the TCS system is the Tactical Microwave Radio (TMR) radio set which is operated in the frequency band of 1.71 to $1.84 \mathrm{GHz}$. Nominal range of this equipment is $15 \mathrm{~km}$ depending on terrain. 


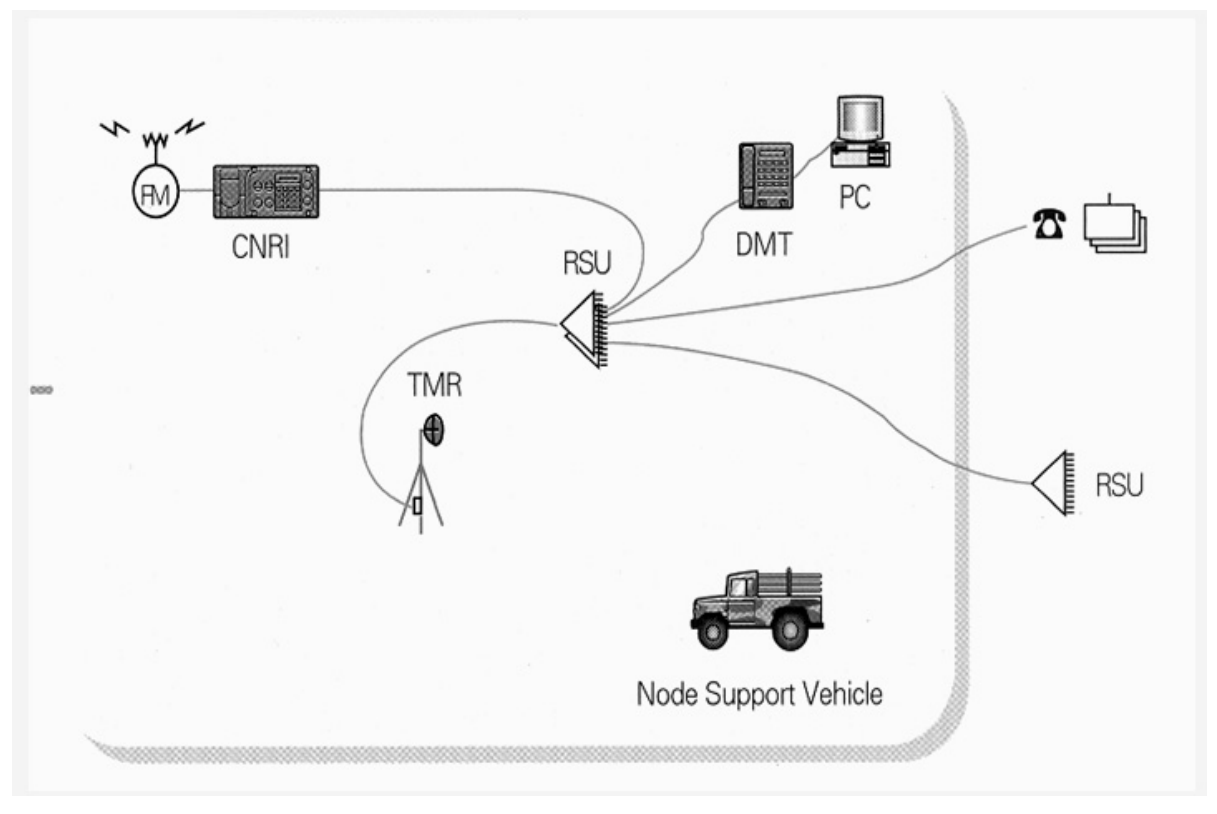

Fig. 2. Unit Level Switch (ULS)

\section{Modeling and simulation results}

The system performance in terms of this MOE factor is best accessed by the use of modeling and simulation techniques. In this section we shall analyze the average link delay versus network size and arrival rate which is related to GOS factors. The network model used in this study is a circuit-switched system overlaid with a packet-switch grid mobile network providing voice and data communications for division/corps area with a maximum of four links. All the links which have the same transmission bandwidth are capable of transmission in both directions. For input traffic at a given source node, the transit traffic at any other node is dependent only on the relative position of this node with respect to the source node. The common channel signaling (CCS) method is used between nodes, and the EUROCOM recommended message format is assumed. CCS are dedicated signaling method that transport both circuit associated (call set up) and non-circuit-associated (transaction capabilities) signaling messages, separate from the voice and data path. Network outages affecting CCS, caused by transmission equipment failure and/or traffic congestion can severely disrupt the delivery of communication services for command and control ${ }^{[7]}$. In anticipation of the possibility of such outages, survivability considerations play a prominent role in the design of military networks. Among the survivability techniques commonly applied to the design of CCS network, there are architectural diversity techniques that consist of deploying CCS network elements arranged in grid or mesh topology to achieve a high level of redundancy. The trunk group in this modeling contains $32 \mathrm{speech} /$ data channels with a $32 \mathrm{kbit} / \mathrm{s}$ common channel. One channel is for all routing functions, one for time synchronization, and the other one for data packets. The remaining 29 channels are available for tandem switching, enough to furnish a low probability of call blocking estimated to be less than $0.3 \%$.

A call is considered as a basic unit of circuit-switched traffic, and each call originating from $\mathrm{S}$ is destined to $\mathrm{D}$. The call arrival process is assumed to be Poisson with rate $\lambda_{i}$ on the $i$-th link and the call holding time is exponentially distributed with mean $1 / \mu$. The call connection and disconnection times are assumed to be statistically independent. Blocked calls are also assumed to be cleared and do not return ${ }^{[8]}$.

To meet desired performance objectives, we study end-to-end time delay of flood search messages in $m$ by $m$ mesh networks. We assume that the arrival process of search messages at each node is Poisson process with $\lambda$ for every (S, D) node pair where they are independent, identically distributed. And we assume exponential average service time with $1 / \mu$ and independence of traffic statistics in different links. Search messages at each link can be approximated as M/M/1 queuing model by Jackson's Theorem ${ }^{[2,3]}$. For our analysis, let us denote several parameters as follows: $H$ is the number of hops, $1 \leq h \leq[(m-1) / 2]$ where $[f]$ represents the greatest integer less than or equal to $f, n(h)$ is the number of nodes that search message 
travels from source to destination node within $h$ hops, $C$ is the channel capacity at link $i$ and $T$ is the average time delay at each link. For $m$ by $m$ mesh networks, $n(h)$ is given by

$$
n(h)=1+\sum_{k=1}^{h} 4 k=2 h(h+1)+1
$$

The probability that search message finds the destination node, $P_{f}$, is $n(h) / m^{2}$. The search message from the source node is transmitted sequentially to four adjacent nodes, and the search message from other nodes is transmitted to three adjacent nodes. Now, the number of search messages generated by a call, $N_{s m}(h)$, is given by

$$
N_{s m}(h)=3(n(h-1)-1)+4=3 n(h-1)+1
$$

Thus, total traffic flows in CCS (messages/sec), $\gamma$, is $\lambda m^{2} N_{s m}(h)$ and arrival rate at link $i$, is given by

$$
\lambda_{i}=\gamma / 4 m^{2}=\lambda N_{s m}(h) / 4
$$

Now, we obtain the time delay at each link, $t_{d}$,

$$
t_{d}=1 /\left(\mu c-\lambda_{i}\right)
$$

Hence, we obtain

$$
T=\sum_{k=1}^{h} k_{t d} 4 a / n(h)
$$

The system uses a common channel signaling, so one channel is used to setup calls among multiple channels in each link. Each node needs a buffer to store signal packets. The performance measure to be used is end to end time delay. In Fig. 3, average link delay versus network size is plotted when $\lambda=0.1$ and $c=1$. For $m=10$ (network size $=100$ ), average link delay is $50 \mathrm{~ms}$ which is similar to practical value of $58 \mathrm{~ms}$ when measured in the test-bed system. In Fig. 4, average link delay versus arrival rate is plotted when arrival rate changes. For $m=20$, average link delay is less than $50 \mathrm{~ms}$. For $m=40$, average link delay is $100 \mathrm{~ms}$ when arrival rate is 0.6 Erlang and it increases exponentially when arrival rate is larger than 0.6 Erlang. In Fig. 5 , average link delay versus channel capacity is plotted when channel capacity at link i changes. Increasing channel capacity with total offered traffic fixed, average link delay performance becomes better as expected. Also, it is seen that when channel capacity is 30 , average link delay is almost $100 \mathrm{~ms}$ for different network size. This result gives valid reason that the trunk group in our study contains 32 speech/data channels. From these results, we have concluded that flooding algorithm for tactical communication networks provides an efficient alternative to multi-path routing for small to medium sized networks.

\section{Conclusions}

Many countries have platforms equipped with technologies approaching obsolescence or requiring communications and information systems re-architecting; to provide some level of information superiority. The communication system is to provide for information transmission within the system of command. Army officers stress that it is the area systems now being deployed that will provide the backbone of the tactical communications architecture to dominate command and control support. An area trunk network provides a grid or mesh of switching centers deployed to provide coverage of the area of operations. It also provides the basic cost effective capability to evolve to the newer system, wireless communications network. Nodes are interconnected by bearers which are traditionally multi-channel radio relay links in the VHF/UHF or SHF band. Headquarters connect to the nearest trunk node by radio relay and can then have access to any other headquarters that is also connected to the network. 


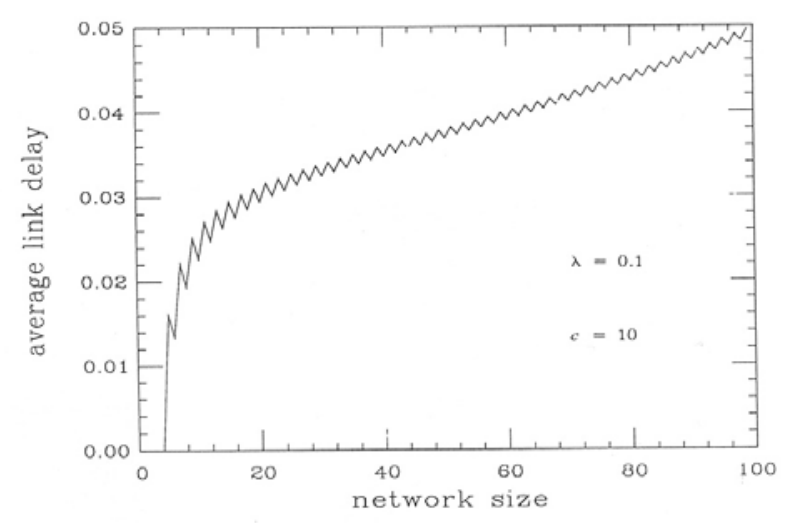

Fig. 3. Average link delay versus network size.

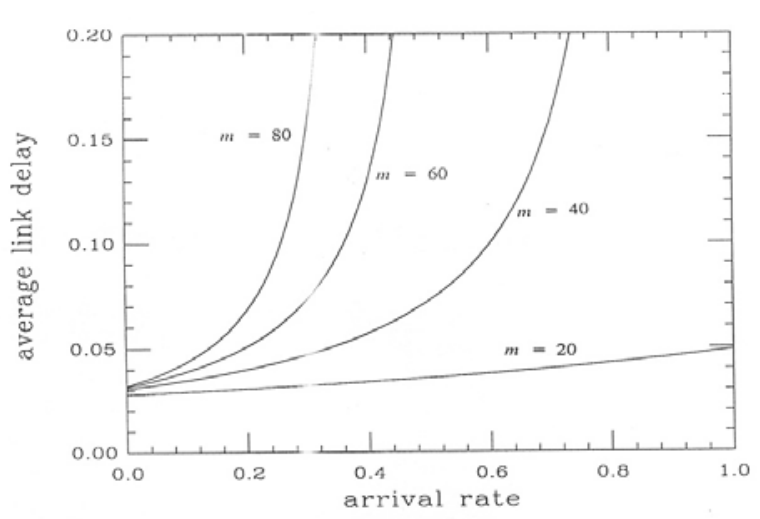

Fig. 4. Average link delay versus arrival rate.

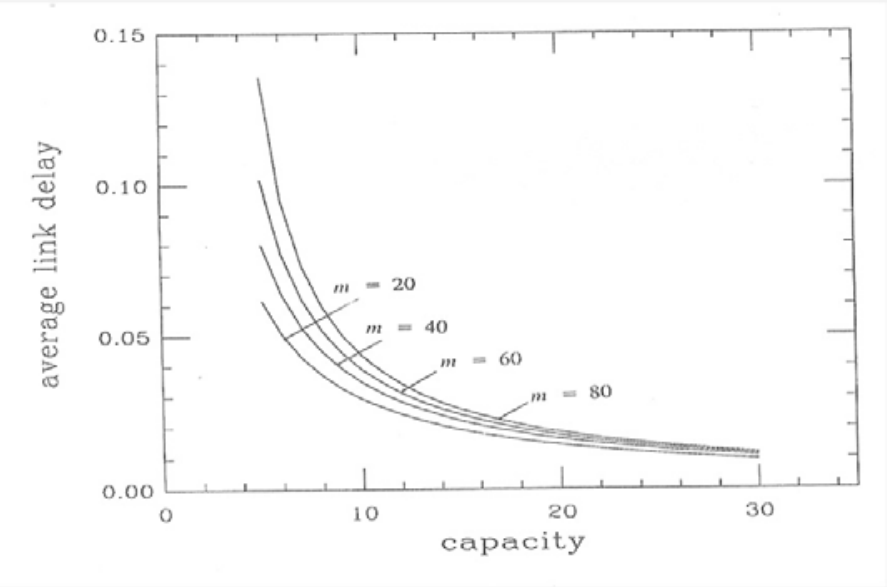

Fig. 5. Average link delay versus capacity.

The system performance in terms of this MOE factor is best accessed by the use of modeling and simulation techniques. This paper has addressed the possible measure of effectiveness (MOE) factors for system design characteristics of tactical communications system. The network model used in this study is a circuitswitched system overlaid with a packet-switch grid mobile network providing voice and data communications for division/corps area with a maximum of four links. Terrestrial Communications System (TCS) architecture for Army is also described. And we proposed a survivable routing algorithm, flood search routing, in tactical grid mobile networks. The primary insight from our study is that survivability considerations play a prominent role in the design of military networks. Among the survivability techniques commonly applied to the design of CCS network, there are architectural diversity techniques that consist of deploying CCS network elements arranged in grid or mesh topology to achieve a high level of redundancy, and that utilizes the flood search routing algorithm for multi-path routing. As a grade of service (GOS) factor, the link delay performance of flood search messages in $\mathrm{m}$ by $\mathrm{m}$ mesh networks is analyzed. We have shown that flooding algorithm provides an efficient alternative to multi-path routing for small to medium sized (i.e. $m=20$ ) networks. We believe that the flood search routing algorithm provides survivability and is a viable option for providing a reliable routing framework in Army Terrestrial Communications System (TCS). The switching network's connectivity and throughput efficiency are further study areas.

\section{References}

[1] Win-t (war-fighter information network-tactical). Www.globalsecurity.org/military/systems/ ground/ win-t.htm.

[2] G. Barrenetxea, etc. Efficient routing with small buffers in dense networks. IEEE ISPN 2005, 2005, $277-284$.

[3] D. Bertsekas, R. Gallager. Data networks, international editions edn. Prentice-Hall, 1992.

[4] C. Duncombe. Integration of commercial routing with flood search routing. IEEE Milcom 1998, 1998, $422-425$. 
[5] D. Goodman, etc. The evolution of untethered communications. in: National Research Council, National Academy Press, Washington, D.C., 1997.

[6] M. Hiroaki, etc. Unidirectional ad hoc routing protocol with area-controlled flooding using overheard neighbor node information. IEEE ISADA'07, 2007, 519-525.

[7] M. Mostrel. Issues on the design of survivable common channel signaling networks. IEEE Journal on selected areas in communications, 1994, 12(3): 526-532.

[8] Y. Park. Design considerations of terrestrial communications system. IEEE IEEM 2007, 2007, 792-795.

[9] M. Ryan, M. Frater. An architectural framework for modern tactical communications systems. Milcom 2000, 2000, 665-670.

[10] N. Sproles. Coming to grips with measures of effectiveness. in: Systems Engineering, The Journal of the International Council on Systems Engineering, vol. 3, John Wiley \& Sons, Inc., New York, 2000, 50-58. No. 1.

[11] D. Vergados, etc. New generation features for tactical wireless communication networks. IEEE VTC 2000, 2000, $542-549$.

[12] A. Ween, N. Tomecko, D. Gossink. Communications architectures and capability improvement evaluation methodology. Milcom 2000, 2000, 988-993. 